well in the countries of Europe today. Only the work of the true evangelist will preserve the essence of the Christian gospel in Africa. In view of the attitude held by the boys in Mr. Musgrove's school, and shared by many 'educated' Africans, missionaries find it necessary to separate the functions of evangelist and secular teacher wherever possible, so that the special nature of the evangelist's work may be properly understood.

\title{
Recherches dans la Côte d'Ivoire
}

M. B. Hol.As, ethnologue du Centre I.F.A.N. de Côte d'Ivoire, a effectué, au cours des mois de mai et juin 1952, une mission ayant pour but l'étude des répercussions qu'exerce le culte de Massa (Alakora en dialecte dioula), depuis le début de I95 I, sur les institutions coutumières anciennes. Ce nouveau culte, pénétrant avec une tapidité et une virulence prodigieuses dans les régions avoisinantes de la Haute-Volta et du Soudan Français, prend alors en général un caractère exclusif et combat vigoureusement les religions autochtones. Ainsi dans la Subdivision Centrale du Cercle de Korhogo (dans le nord de la Côte d'Ivoire), le Massa a-t-il même réussi à exercer une influence remarquable sur l'organisation du poro qui, on le sait, représente - ou représentait jusqu'ici - une base séculaire de toute la vie socioreligieuse des Sénoufo non-islamisés.

Dans cette dernière région, l'avènement du Massa ayant amené l'abandon presque total des inventaires cultuels anciens, une collection de plus de 700 pieces d'intérêt documentaire considérable a pu être constituée pour enrichir le Musée du Centre I.F.A.N. à Abidjan.

$L$ 'enquête a mené $M$. Holas jusqu'au foyer de diffusion du nouveau culte qui se situe dans le Cercle de San au Soudan Français où de précieuses informations ont été recueillies directement auprès du fondateur du Massa, chef suprême du culte, un Minianka nommé Peni Dembélé.

Un compte-rendu préliminaire sur ce nouveau mouvement religieux est en préparation.

Par ailleurs, au cours de la même mission, des observations ont été faites dans la région de Gaoua (Haute-Volta) sur les cérémonies finales du dyoro, fête septennale des initiations lobibirifor, et une riche documentation photographique et cinématographique a été constituée.

\section{Editions de disques du Musée de l'Homme}

A LA suite de ses éditions de Madagascar et d'Afrique équatoriale française, le Département d'ethnologie musicale au Musée de l'Homme présente une collection nouvelle de musique du Sahara. Cette collection a été établie par les soins de M. André Schaeffner, maître de recherches au Centre National de la Recherche scientifique, et de M. Gilbert Rouget, du Département d'ethnologie musicale. Les documents utilisés furent recueillis en 1948 sous la direction de M. Henri Lhote et enregistrés par M. Alain Joset, mort depuis au cours d'une mission. Le matériel d'enregistrement avait été prêté par la Radiodiffusion française, et c'est grâceà l'obligeant concours de l'Office de la Recherche scientifique Outre-Mer que cette édition a pu être entreprise.

Cette édition se compose de 14 disques double-face de $25 \mathrm{~cm}$. Elle contient so pièces de musique, représentatives du répertoire de plusieurs populations : Touaregs du Hoggar, de l'Adrat des Iforas, du Tamasna, etc. . . .; femmes Orféla du Fezzan, réfugiées au Hoggar; femmes Harratines, originaires de l'oasis d'In-Salah; soit, à la fois, des Berbères, des Arabes, des métis de Berbères et de Noirs du Soudan. Ce n'est pas le moindre intérêt de cette collection que de permettre de comparer les musiques de plusieurs races ou tribus vivant sur le même territoire. Comme dans les précédentes éditions de Madagascar et d'Ogooué-Congo l'on a emprunté abondamment au répertoire vocal, le chant, en solo ou en chour, constituant l'un des sommets de la musique africaine et étant relativement peu 